\title{
Characterization of bijective digitized rotations on the hexagonal grid
}

\author{
Kacper Pluta - Tristan Roussillon - David Courjolly • Pascal \\ Romon • Yukiko Kenmochi • Victor Ostromoukhov
}

the date of receipt and acceptance should be inserted later

\begin{abstract}
Digitized rotations on discrete spaces are usually defined as the composition of a Euclidean rotation and a rounding operator; they are in general not bijective. Nevertheless, it is well known that digitized rotations defined on the square grid are bijective for some specific angles. This infinite family of angles has been characterized by Nouvel and Rémila and more recently by Roussillon and Cœurjolly. In this article, we characterize bijective digitized rotations on the hexagonal grid using arithmetical properties of the Eisenstein integers.
\end{abstract}

\section{Introduction}

Rotations defined on discrete spaces are simple yet crucial operations in many image processing applications involving 2D data: for example, template matching [5], object tracking [25] and halftoning and printing $[19,20]$. While rotations defined on $\mathbb{R}^{2}$ are isometric and bijective, their digitized cousins, in general, do not preserve distances and are not

Kacper Pluta and Yukiko Kenmochi

Université Paris-Est, LIGM, CNRS-ESIEE Paris, France

Kacper Pluta and Pascal Romon

Université Paris-Est, LAMA, France

Tristan Roussillon, David Cœurjolly and Victor Ostromoukhov

Université de Lyon, CNRS, LIRIS, UMR5205, F-69622, France bijective. Over last 20 years, digitized rotations on the square grid have attracted more attention than ones defined on the hexagonal grid. Indeed, the square grid is predominant over other grids in fields such as image processing, mostly because of its common use by image acquisition devices, even though it is burdened with fundamental topological problems - one has to choose between different connectivity relations for objects of interest and their complements $[12,13]$.

On the contrary, the hexagonal grid is free from these problems since it possesses the following properties: equidistant neighbors - each hexagon has six equidistant neighbors; uniform connectivity - there is only one type of connectivity [15, 24]. Besides, the famous honeycomb conjectureproved by Hales [8] — states that the regular hexagonal grid is the best way to divide Euclidean plane into regions of equal area with the least total perimeter. The hexagonal grid has also been shown to provide better solutions to other problems such as reconstruction of continuous signals from sampled data [2,3] and edge detection [14] to name some. We refer readers who are interested in an exhaustive comparison between image processing tasks with respect to the hexagonal and square grids to the book by Middleton and Sivaswamy [15].

Concerning the characterization of the bijective digitized rotations on the square grid, the most important studies are the following: Andres and 
Jacob were the first to give a sufficient condition for bijectivity [11]; Nouvel and Rémila prove its necessity [17] using their framework introduced in [16]; more recently Roussillon and Cœurjolly provided another proof which is based on the arithmetical properties of Gaussian integers [23]. It is also worth to mention that Pluta et al. used an extension of the framework proposed originally by Nouvel and Rémila [16], to characterize bijective digitized rigid motions [21].

Studies related to digitized rotations on the hexagonal grid are less numerous: Her in [10]while working with the hexagonal grid represented by cube coordinate system in 3D-showed how to derive a rotation matrix such that it is simpler than a 3D rotation matrix obtained in a direct way; Pluta et al. in [22] provided a framework to study local alterations of discrete points on the hexagonal grid under digitized rigid motions. They also characterized Eisenstein rational rotations on the hexagonal grid which, together with their framework, allowed them to compare the loss of information induced by digitized rigid motions with the both grids . Indeed, it is shown in [22] that, on average, digitized rigid motions defined on the hexagonal grid lose less information than their counterparts defined with respect to the square grid.

In this article, we consider an approach similar to the one proposed by Roussillon and Cœurjolly [23], based on complex numbers. Indeed points in $\mathbb{Z}^{2}$ can be identified with Gaussian integers, and rational rotations correspond to multiplications by complex numbers of unit modulus with rational real and imaginary parts. For the hexagonal lattice, we use Eisenstein integers instead of Gaussian integers [4,9]. First, we discuss the density of rational rotations on the hexagonal grid, which were characterized in [22]. That is similar to the study of rational rotations on the square grid - angles given by right triangles of integer sides [1]. Then, we prove that there exist two subsets of Eisenstein integers yielding bijective digitized rotations. This differs from the square case, where only one subset is involved. We also show that bijective digitized rotations on the hexagonal grid are more numerous than their counterparts on the square grid for angles relatively close to multiple of $\frac{\pi}{3} k, k \in \mathbb{Z}$ angle.

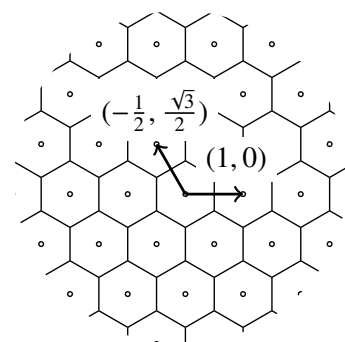

(a)

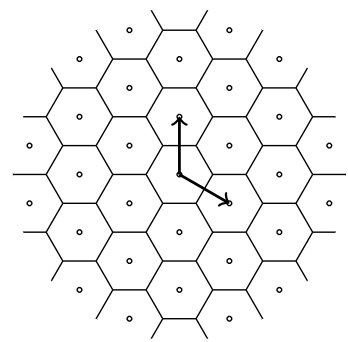

(b)
Fig. 1 Two variations of hexagonal grids, called pointy topped (a) and flat topped (b) hexagonal grids which are equivalent up to rotation by $(2 k+1) \frac{\pi}{6}, k \in \mathbb{Z}$. Arrows represent the bases of the underlying lattices

\section{Eisenstein integers}

A hexagonal grid $\mathcal{H}$ is a grid formed by a tessellation of the complex plane $\mathbb{C}$ by hexagons of side length $\frac{1}{\sqrt{3}}$. Figure 1 shows two examples of such hexagonal grids. Hereafter, without lack of generality, we consider pointy topped hexagonal grid (see Figure 1). In such a setting we consider centroids of hexagons, i.e., the points of the underlying lattice $\Lambda:=\mathbb{Z} \oplus \mathbb{Z} \omega$, where $\omega:=-\frac{1}{2}+\frac{\sqrt{3}}{2} i$, is a solution of $\omega^{2}+\omega+1=0$. Thus $\mathcal{H}$ is the Voronoi tessellation associated with the lattice $\Lambda$ in $\mathbb{C}$. The elements of $\Lambda$ are called Eisenstein integers [4,9]; they are the complex numbers of the form

$$
\alpha:=a+b \omega,
$$

where $a, b \in \mathbb{Z}$.

\subsection{Properties of Eisenstein integers}

The Eisenstein integers form a Euclidean ring $\mathbb{Z}[\omega]$ in $\mathbb{C}$; in particular they possess similar properties to the ordinary integers: division, prime factorization and greatest common divisor are well defined. Let us consider $\alpha:=a+b \omega \in \Lambda$;

- The conjugate of $\alpha$ is given as $\bar{\alpha}=(a-b)-b \omega$.

- The squared modulus of $\alpha$ is given as $|\alpha|^{2}:=$ $\alpha \cdot \bar{\alpha}=a^{2}-a b+b^{2}$.

- The units of $\Lambda$ are $\gamma:=\{ \pm 1, \pm \omega, \pm \bar{\omega}\}$, where $\bar{\omega}=\omega^{2}$.

- $\alpha$ is divisible by $\beta$ if there exists $\phi \in \Lambda$ such that $\alpha=\beta \cdot \phi$. 
- Given $\alpha, \beta \in \Lambda$, a greatest common divisor $\operatorname{gcd}(\alpha, \beta)=\phi \in \Lambda$ is defined as a largest Eisenstein integer (up to multiplications by units) that divides both $\alpha$ and $\beta$; every common divisor of $\alpha$ and $\beta$ also divides $\phi$. By a largest Eisenstein integer, we mean one of a largest modulus.

- $\alpha$ is said to be an Eisenstein prime if its divisors are only of the form $v \cdot \alpha$ such that $v \in \Upsilon$.

Moreover, as for the ordinary complex numbers, the product of two Eisenstein integers has a geometric interpretation. Let $\alpha, \beta \in \Lambda$, then $\alpha \cdot \beta$ is equal to a combination of a rotation by angle $\theta:=\tan ^{-1}\left(\frac{\sqrt{3} b}{2 a-b}\right)$ and the scaling by $|\alpha|$. Note that $\sin \theta=\frac{\sqrt{3} b}{2|\alpha|}$ and $\cos \theta=\frac{2 a-b}{2|\alpha|}$.

\subsection{Eisenstein triples}

Eisenstein triples $(a, b, c) \in \mathbb{Z}^{3}$ are triples of integers such that

$$
|\alpha|^{2}=a^{2}-a b+b^{2}=c^{2} .
$$

Thanks to symmetry, hereafter, we consider only positive primitive Eisenstein triples, i.e., $0<a<$ $c<b, \operatorname{gcd}(a, b, c)=1$ and either $a+b+c \not \equiv 0$ $(\bmod 3)$ or $2 b-a+c \not \equiv 0(\bmod 3)$ [7]. The later condition, is that we exclude triples which lead to rotations by $k \frac{\pi}{3}, k \in \mathbb{Z}$, e.g., $(1,1,1)$. Note that, if $\operatorname{gcd}(a, b, c)=d \in \mathbb{Z}^{+}$the rotation by $a+b \omega$ reduces to that given by the primitive Eisenstein triple $\frac{1}{d}(a, b, c)$, with the scale factor. Let us then denote the set of positive primitive Eisenstein triples by $E_{\rho}^{+}$and consider the subset

$$
\Lambda_{\rho}^{+}:=\left\{\alpha:=a+b \omega \in \Lambda \mid\left(a, b, \sqrt{a^{2}-a b+b^{2}}\right) \in E_{\rho}^{+}\right\} .
$$

A part of $\Lambda_{\rho}^{+}$is illustrated by Figure 2. Moreover, we denote by $E_{\rho}$ and $\Lambda_{\rho}$, respectively, the set of primitive Eisenstein triples and a subset of $\Lambda$ which is defined in a similar way to $\Lambda_{\rho}^{+}$.

Lemma 1 ([7]) Positive integers $a, b$ and $c$ form a pair of primitive Eisenstein triples $(a, b, c)$ and $(b-a, b, c)$, if and only if there exist $s, t \in \mathbb{Z}, 0<$ $s<t, \operatorname{gcd}(s, t)=1$ and $t-s \not \equiv 0(\bmod 3)$, such

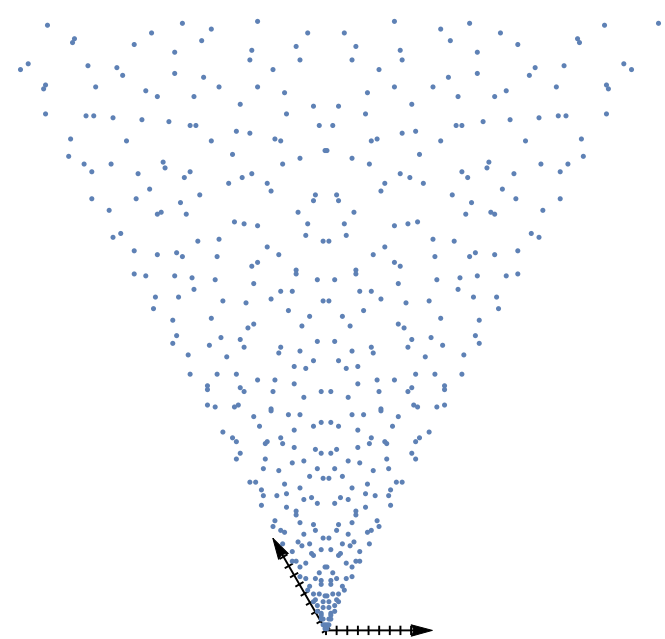

Fig. 2 Visualization of $\Lambda_{\rho}^{+}$for $0<a, b \leq 2000$. Note that the axes were scaled for a better visualization effect and the distance between two consecutive axes' ticks is 30

that

$$
\begin{aligned}
& a=s^{2}+2 s t, \\
& b=t^{2}+2 s t, \\
& c=s^{2}+t^{2}+s t .
\end{aligned}
$$

For the proof, see the discussion provided by Gilder in [6].

Notice that the union of triangles of side lengths $(a, b, c) \in \mathbb{Z}^{3}$ and $(b-a, b, c) \in \mathbb{Z}^{3}$ is equal to an equilateral triangle of the integer side length equal to $b$ (see Figure 3). For more information about Eisenstein integers we encourage the reader to look into $[6,7]$.

Furthermore, we state the following results related to factoring of primitive Eisenstein integers and used in the later sections.

Lemma 2 Let $\alpha$ be an Eisenstein integer such that $\alpha$ is not the product of an integer and an Eisenstein unit. Then $\alpha=\bar{\alpha} \cdot v$ where $v \in \Upsilon$, if and only if $|\alpha|^{2} \equiv 0(\bmod 3)$.

Proof Solving $\alpha=\bar{\alpha} \cdot v$ where $v \in \Upsilon$, is equivalent to solving $\alpha^{2}=|\alpha|^{2} v$, whose solutions (in $\mathbb{C})$ are $\alpha= \pm|\alpha| \sqrt{v}$, where $\sqrt{v}$ means any root. Two possibilities occur: (i) $v \in\{1, \omega, \bar{\omega}\}$, which are squares in $\Lambda$; this is the first case in the Lemma; (ii) 


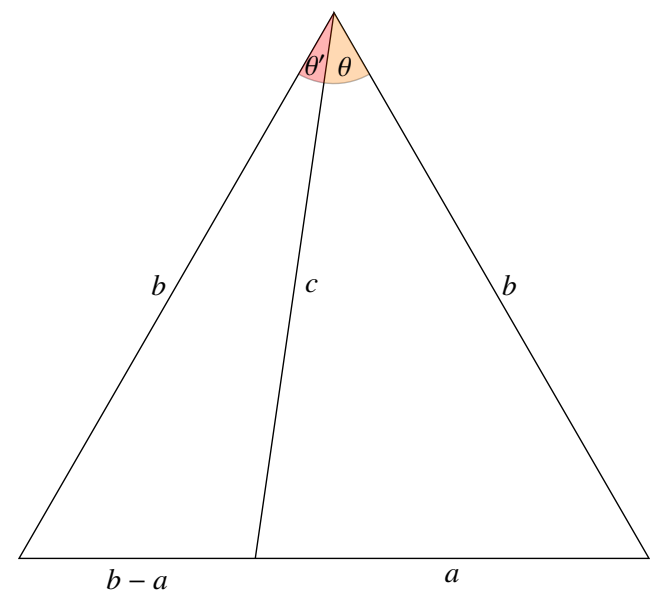

Fig. 3 Geometric interpretation of a pair of Eisenstein triples, $(a, b, c)$ and $(b-a, b, c)$, where $\theta=\tan ^{-1}\left(\frac{\sqrt{3} a}{2 b-a}\right)$ and $\theta^{\prime}=\tan ^{-1}\left(\frac{\sqrt{3}(b-a)}{b+a}\right)$

$v \in\{-1,-\omega,-\bar{\omega}\}$, whose roots are not in $\Lambda$ but in $\Lambda / \sqrt{3}$; in order for $\alpha$ to be an Eisenstein integer, its modulus $|\alpha|$ must contains a $\sqrt{3}$ factor.

Lemma 3 For a given $\alpha:=a+b \omega \in \Lambda_{\rho}^{+}$, considering $\gamma:=(s+t)+t \omega \in \Lambda$ where $s$ and $t$ are generators of $(a, b, c)$ (see Lemma 1), we have

$$
\begin{aligned}
& \alpha=\gamma \cdot \gamma, \\
& |\alpha|=c=\gamma \cdot \bar{\gamma}, \\
& |\gamma|^{2} \not \equiv 0 \quad(\bmod 3), \\
& \operatorname{gcd}(\gamma, \bar{\gamma})=1, \\
& \operatorname{gcd}(\alpha, c)=\gamma .
\end{aligned}
$$

Proof Equations (1-2) are direct consequences of the parameterization given in Lemma 1.

For Statement (3), let us suppose that there exists $v \in \Lambda$ such that $\gamma=\mu \nu$ with $|\mu|^{2}=3$ and $\mu \in \Lambda$. Since the squared modulus is multiplicative, $|\gamma|^{2}=(|\mu \| v|)^{2}$ and thus $c=3|v|^{2}$, which means that 3 divides $c$. From [7, Theorem 1], we know that 3 does not divide $c$ for a valid prime Eisenstein triple, which contradicts the hypothesis that $v$ exists.

To prove (4), let us consider prime factors $\left\{\pi_{i}\right\}_{n}$ of $\gamma$ (resp. $\left\{\pi_{i}^{\prime}\right\}_{n}$ of $\bar{\gamma}$ ). We have $\gamma=\pi_{1} \pi_{2} \ldots \pi_{n}$ (resp. $\bar{\gamma}=\bar{\pi}_{1} \bar{\pi}_{2} \ldots \bar{\pi}_{n}$ ). Such prime factors are uniquely defined up to their associates. From (3) and Lemma 2, prime factor decomposition of $\gamma$ and $\bar{\gamma}$ have no common factors (beside units) and thus $\operatorname{gcd}(\gamma, \bar{\gamma})=1$.

Statement (5) follows from Equations (1-2) and (4).

\section{Digitized rotations}

Given a complex number $\alpha \in \mathbb{C}$, rotations on $\mathbb{C}$ are defined as

$\begin{aligned} \mathcal{U}^{\alpha}: & \mathbb{C} \rightarrow \mathbb{C} \\ x & \mapsto \frac{\alpha \cdot x}{|\alpha|} .\end{aligned}$

According to Equation (6), we generally have $\mathcal{U}^{\alpha}(\Lambda) \nsubseteq \Lambda$. As a consequence, in order to define digitized rotations as maps from $\Lambda$ to $\Lambda$, we commonly apply rigid motions on $\Lambda$ as a part of $\mathbb{C}$ and then combine the results with a digitization operator. To define a digitization operator on $\Lambda$, let us first define, for a given rotation and scaling factor $\phi \in \Lambda$ and for all $\kappa \in \Lambda$, the hexagonal cell

$$
C_{\phi}(\kappa):=\left\{\begin{array}{l|r}
x \in \mathbb{C} \mid \begin{array}{rr}
\forall v \in \Upsilon^{+},(\|x-\kappa\| \leq\|x-\kappa+v \cdot \phi\| \\
\wedge & \|x-\kappa\|<\|x-\kappa-v \cdot \phi\|)
\end{array}
\end{array}\right\},
$$

where $\Upsilon^{+}:=\{1, \omega,-\bar{\omega}\}$. Figure 4 illustrates some hexagonal cells with different $\phi$. Finally, we define the digitization operator as a function $\mathcal{D}: \mathbb{C} \rightarrow \Lambda$ such that $\forall x \in \mathbb{C}, \exists ! \mathcal{D}(x) \in \Lambda$ and $x \in \mathcal{C}_{1}(\mathcal{D}(x))$. We define then digitized rigid motions as $U^{\alpha}=$ $\mathcal{D} \circ \mathcal{U}_{\mid \Lambda}^{\alpha}$.

Due to the behavior of $\mathcal{D}$ that maps $\mathbb{C}$ onto $\Lambda$, digitized rotations $U^{\alpha}$ are, most of the time, nonbijective (see Figure 5).

\section{Bijectivity of digitized rotations}

\subsection{Set of remainders}

Let us compare the rotated lattice $\mathcal{U}^{\alpha}(\Lambda)$ with $\Lambda$. The digitized rotation $U^{\alpha}:=\mathcal{D} \circ \mathcal{U}^{\alpha}$ is bijective if and only if $\forall \lambda \in \Lambda \exists ! \kappa \in \Lambda$ such that $\mathcal{U}^{\alpha}(\kappa) \in C_{1}(\lambda)$. This is equivalent to the "double" 
surjectivity relation, used by Roussillon and Cœurjolly [23]:

$$
\left\{\begin{array}{l}
\forall \lambda \in \Lambda, \exists \kappa \in \Lambda, \mathcal{U}^{\alpha}(\kappa) \in C_{1}(\lambda) \\
\forall \kappa \in \Lambda, \exists \lambda \in \Lambda, \lambda \in C_{\frac{\alpha}{|\alpha|}}\left(\mathcal{U}^{\alpha}(\kappa)\right)
\end{array}\right.
$$

provided that $\kappa$ and $\lambda$ in the first and the second conditions of Formula (7) are the same (see Figure 6).

Instead of studying the whole source and target spaces, we study the set of remainders defined by the map

$$
\begin{aligned}
S_{\alpha}: \Lambda \times \Lambda & \rightarrow \mathbb{C} \\
(\kappa, \lambda) & \mapsto \frac{\kappa \cdot \alpha}{|\alpha|}-\lambda .
\end{aligned}
$$

Then, (7) can be rewritten as

$\left\{\begin{array}{l}\forall \lambda \in \Lambda, \exists \kappa \in \Lambda, S_{\alpha}(\kappa, \lambda) \in C_{1}(0) \\ \forall \kappa \in \Lambda, \exists \lambda \in \Lambda, S_{\alpha}(\kappa, \lambda) \in C_{\frac{\alpha}{|\alpha|}}(0),\end{array}\right.$

provided that $S_{\alpha}(\Lambda, \Lambda) \cap \bigodot_{1}(0)=S_{\alpha}(\Lambda, \Lambda) \cap \bigodot_{\frac{\alpha}{|\alpha|}}(0)$. In other words

$S_{\alpha}(\Lambda, \Lambda) \cap\left(\left(C_{1}(0) \cup C_{\frac{\alpha}{|\alpha|}}(0)\right) \backslash\left(C_{1}(0) \cap C_{\frac{\alpha}{|\alpha|}}(0)\right)\right)=\varnothing$.

\subsection{Eisenstein rational rotations}

The key to understand the conditions that ensure the bijectivity of $U^{\alpha}$ is the structure of the images of $S_{\alpha}(\Lambda, \Lambda)$, i.e.,

$$
\mathcal{L}:=\mathbb{Z} \frac{\alpha}{|\alpha|} \oplus \mathbb{Z} \frac{\alpha \cdot \omega}{|\alpha|} \oplus \mathbb{Z} \oplus \mathbb{Z} \omega
$$

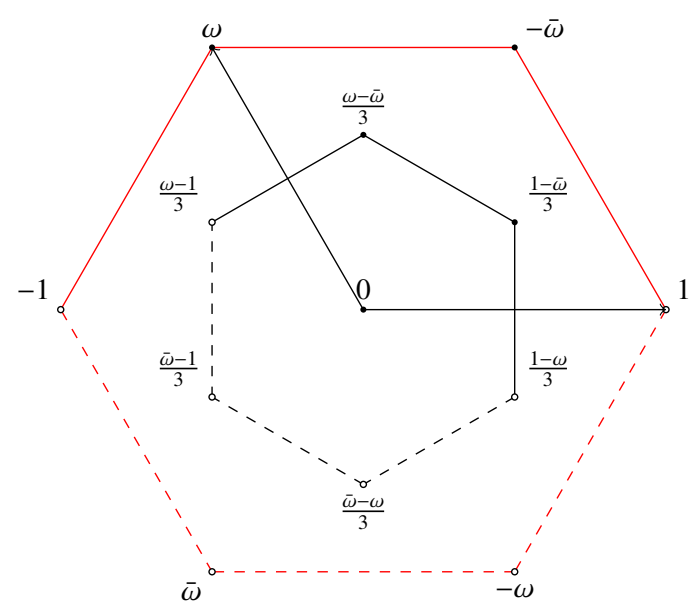

Fig. 4 Visualization of hexagonal cells: $\mathcal{C}_{1}(0)$ (in black) $e_{(2+\omega)}(0)$ (in red). Dashed lines and white circles-which represents points—are not included in hexagonal cells

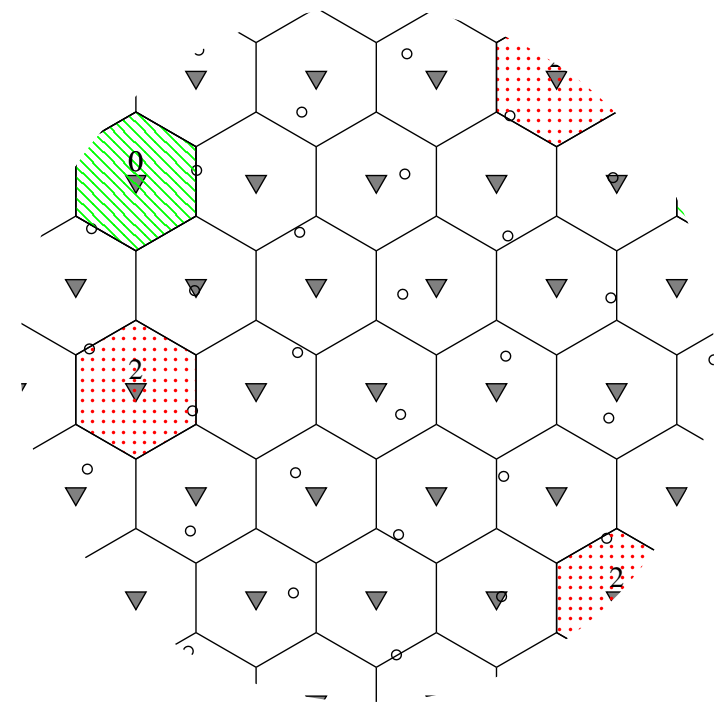

Fig. 5 Examples of three different point mappings: digitization cells which contain zero or two points of $\mathcal{U}^{\alpha}(\Lambda)$ are marked by green lined and red dotted patterns, respectively. Moreover, such cells have labels which correspond to a number of the points. White dots indicate the positions of the images of the points of the initial set $\Lambda$ under $\mathcal{U}^{\alpha}$

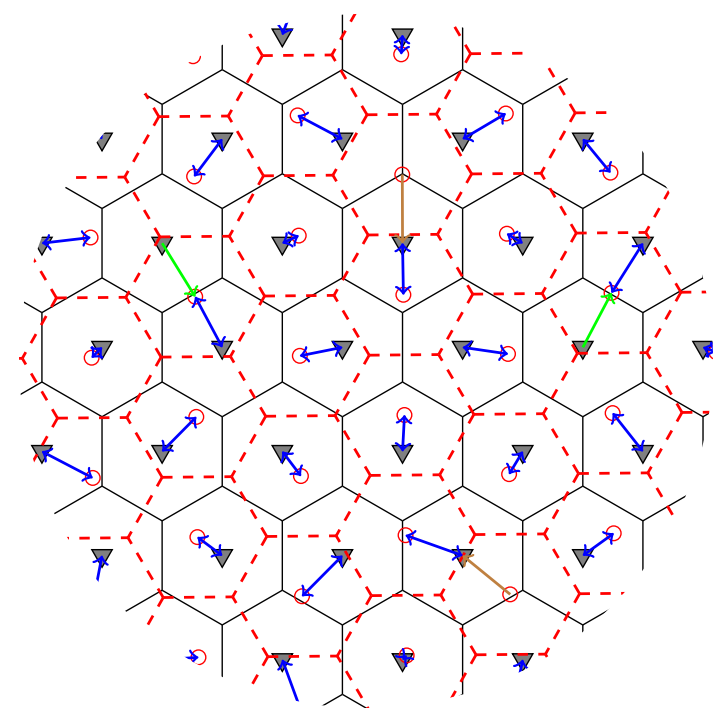

Fig. 6 Visualization of a mapping between $\Lambda$ and $\mathcal{U}^{\alpha}(\Lambda)$. Arrows which corresponds to bijective and non-bijective mappings are marked in blue and green (resp. brown), respectively. (see Formula (7))

For this reason, we start by looking at $\mathcal{L}_{\mathcal{L}}$, and discuss its density. In the case of the square grid, it is known that the equivalent of $\mathcal{G}_{\mathcal{L}}$ is a lattice if and only if cosine and sine are rational numbers, i.e., 
rotations are given by primitive Pythagorean triples $[16,18,21]$. When on the contrary cosine or/and sine are irrational, $\mathcal{L}$ is an infinite and dense set $[16,18,21]$.

In [22] we proved that in the hexagonal grid case a similar result is obtained for rotations corresponding to Eisenstein integers. We shall say that a rotation $\mathcal{U}^{\alpha}$ is Eisenstein rational ${ }^{1}$ if $\alpha$ is given by a Eisenstein triple and then we have the following result [22, Proposition 9].

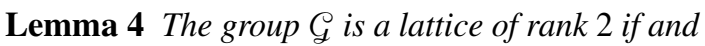
only if $\alpha \in \Lambda$

When $C_{\mathcal{l}}$ is not a lattice, then we have the following.

Lemma 5 Whenever $\mathcal{G}_{\mathcal{L}}$ is not a lattice the corresponding digitized rotation is not bijective.

Proof Since $\mathcal{G}_{2}$ is dense, there exist $\mu, \mu^{\prime} \in \mathcal{G} \cap$ $C_{1}(0)$ such that $S_{\alpha}(\kappa, \lambda)=\mu$ and $S_{\alpha}(\kappa+1, \lambda)=\mu^{\prime}$. Then, we have $\mu+\frac{\alpha}{|\alpha|}=\mu^{\prime}$ and $\frac{\kappa \cdot \alpha}{|\alpha|}, \frac{(\kappa+1) \cdot \alpha}{|\alpha|} \in C_{1}(\lambda)$ for some $\kappa, \lambda$. On the contrary, if this is not the case, then $\mathcal{L}_{\text {is not dense. }}$

We hereafter, consider only Eisenstein rational rotations for the bijectivity characterization.

\subsection{Density of Eisenstein rational rotations}

Let us focus on the density of rational rotations, in general. In the case of rational rotations given by Pythagorean triples it is known that they are dense [1]. On the other hand, in the case of Eisenstein rational rotations we provide the following result.

Lemma 6 Eisenstein rational rotations are dense.

Proof Let $\theta \in \mathbb{R}$ be any angle. Then there are $u, v \in$ $\mathbb{R}$ such that $\theta:=\arg (u+v \omega)$. For a rational rotation to have an angle close to $\theta$, we need $\alpha=a+b \omega \in \Lambda_{\rho}$ to satisfy $\frac{b}{a} \approx \frac{v}{u}$. The same reasoning holds for the square roots of $\alpha$ (see Lemma 3). Hence, we might as well work with $\gamma=(s+t)+t \omega$, and prove that the ratio $\frac{s+t}{t}$ takes a dense set of values

\footnotetext{
1 Note that the relevant notion in hexagonal geometry is not that cosine and sine are rational numbers.
}

in $\mathbb{R}$. Equivalently, we prove that any number $x$ can be approximated with arbitrary precision by a ratio $s / t$ where $s, t \in \mathbb{Z}, \operatorname{gcd}(s, t)=1$ and $s-t \not \equiv$ $0(\bmod 3)$. The first constraint is satisfied by the rational approximations of a real number. There remains to show that we can choose $s, t \in \mathbb{Z}$ so that $s-t \not \equiv 0(\bmod 3)$. Without loss of generality, we restrict to denominators $t$ that are prime; since these can be chosen arbitrarily large, the approximation property still holds.

Now consider $s / t,(s+1) / t$ and $(s-1) / t$. For $t$ large enough, they are all very close to $x \in \mathbb{R}$, so approximation is not an issue. Among these three numerators, only one can be equal to $0 \bmod 3$, and we consider the two remaining ones. Finally, we note that the two numerators cannot be multiples of $t$, because their difference-which is equal to 1 or 2-would also be multiple of $t$.

We recall that any Eisenstein rational rotation which is not given by a primitive Eisenstein triple reduces to such, i.e. if $\alpha \notin \Lambda_{\rho}, \exists ! \alpha^{\prime} \in \Lambda_{\rho}$, such that the corresponding rotations are same.

\subsection{Reduced set of remainders}

Working in the framework of Eisenstein rational rotations defined by $\alpha \in \Lambda_{\rho}$, allows us to turn to Eisenstein integers as $|\alpha| \mathcal{L}_{\mathcal{C}} \subset \Lambda$. For the reason that Eisenstein integers are nicer to work with, we do scale $\mathcal{G}_{\mathcal{L}}$ by $|\alpha|$. Similarly to the former discussion, after scaling $\mathcal{C}_{\mathcal{C}}$ by $|\alpha|$, we consider the finite set of remainders, obtained by comparing the lattice $|\alpha| \mathcal{U}^{\alpha}(\Lambda)$ with the lattice $|\alpha| \Lambda$, and applying the scaled version of the map $S_{\alpha}$ defined as

$$
\mid \begin{aligned}
\check{S}_{\alpha}: \Lambda \times \Lambda & \rightarrow \Lambda \\
(\kappa, \lambda) & \mapsto \kappa \cdot \alpha-|\alpha| \lambda .
\end{aligned}
$$

Indeed, Formula (8) is rewritten by

$$
\left\{\begin{array}{l}
\forall \lambda \in \Lambda \exists \kappa \in \Lambda, \check{S}_{\alpha}(\kappa, \lambda) \in C_{|\alpha|}(0) \\
\forall \kappa \in \Lambda \exists \lambda \in \Lambda, \check{S}_{\alpha}(\kappa, \lambda) \in C_{\alpha}(0) .
\end{array}\right.
$$

Let us consider $\gamma \in \Lambda$ such that $\alpha=\gamma^{2} \in \Lambda_{\rho}^{+}$ and $|\alpha|=\gamma \cdot \bar{\gamma}$ (see Lemma 3). We then see that Formulae (9) and (10) are multiples of $\gamma$. Since 
division by $\gamma$ removes the common multiple while ensuring results to stay in $\Lambda$, let us define

$$
\mid \begin{aligned}
S_{\gamma}^{\prime}: \Lambda \times \Lambda & \rightarrow \Lambda \\
(\kappa, \lambda) & \mapsto \kappa \cdot \gamma-\bar{\gamma} \cdot \lambda
\end{aligned}
$$

Then, the bijectivity of $U^{\alpha}$ is ensured when

$\left\{\begin{array}{l}\forall \lambda \in \Lambda, \exists \kappa \in \Lambda, S_{\gamma}^{\prime}(\kappa, \lambda) \in C_{\bar{\gamma}}(0) \\ \forall \kappa \in \Lambda, \exists \lambda \in \Lambda, S_{\gamma}^{\prime}(\kappa, \lambda) \in C_{\gamma}(0),\end{array}\right.$

provided that $S_{\gamma}^{\prime}(\Lambda, \Lambda) \cap C_{\bar{\gamma}}(0)=S_{\gamma}^{\prime}(\Lambda, \Lambda) \cap C_{\gamma}(0)$ $\Leftrightarrow S_{\gamma}^{\prime}(\Lambda, \Lambda) \cap\left(\left(C_{\gamma}(0) \cup C_{\bar{\gamma}}(0)\right) \backslash\left(C_{\gamma}(0) \cap C_{\bar{\gamma}}(0)\right)\right)=\varnothing$.

\section{Characterization of bijective digitized rotations}

In this section we provide sufficient and necessary conditions for bijectivity of $U^{\alpha}$. Our goal is to prove that the bijectivity of $U^{\alpha}$ is ensured if and only if $\alpha$ is given by a primitive Eisenstein triple such that its generators $s, t$ are of the form $s>0, t=s+1$ or $s=1, t>1$.

Before going into the main discussion of this section, we note that the cyclic order of the vertices of $C_{\gamma}(0)$ and that of $C_{\bar{\gamma}}(0)$ is invariable. It is due to the fact that $|\gamma|=|\bar{\gamma}|, \mathcal{U}^{\gamma}=\mathcal{U}^{-\bar{\gamma}}$ and $0<s<t$. Also, the vertices of the hexagonal cells $C_{\gamma}(0)$ and $C_{\bar{\gamma}}(0)$ are not in $\Lambda$. Indeed, the vertices of $C_{1}(0)$ are not in $\Lambda$ (see Figure 7) and $|\gamma|^{2}$ (resp. $|\bar{\gamma}|^{2}$ ) is not a multiple of 3 (see Lemma 3). Therefore, vertices of $C_{\gamma}(0)=\gamma \cdot C_{1}(0)\left(\right.$ resp. $\left.C_{\bar{\gamma}}(0)=\bar{\gamma} \cdot C_{1}(0)\right)$ are not in $\Lambda$. For the simplification of the following discussion we scale the cells $C_{\gamma}(0)$ and $C_{\bar{\gamma}}(0)$ by $2+\omega$ so that the vertices are in $\Lambda$ (see Figure 7).

Lemma 7 If $s \neq 1$ or $t \neq s+1$, then $\exists \phi \in S_{\gamma}^{\prime}(\Lambda, \Lambda)$ such that $\phi \in\left(C_{\bar{\gamma}}(0) \cup C_{\gamma}(0)\right) \backslash\left(C_{\bar{\gamma}}(0) \cap C_{\gamma}(0)\right)$.

Proof Thanks to the symmetry, we focus on one of the vertices of $C_{\bar{\gamma} \cdot(2+\omega)}(0), \bar{\gamma}$. Then we consider the two closest points of $C_{\bar{\gamma} \cdot(2+\omega)}(0) \cap \Lambda$ to $\bar{\gamma}$, namely, $\zeta:=(\bar{\gamma}-1)$ and $\zeta^{\prime}:=(\bar{\gamma}+\omega)$. Note that since $0<s<t$, we need to consider only this case.

We now show that if $t \neq s+1$ or $s \neq 1$ then $\zeta \notin$ $C_{\gamma \cdot(2+\omega)}(0)$ or $\zeta^{\prime} \notin C_{\gamma \cdot(2+\omega)}(0)$. Figure 8 illustrates examples of such a situation.

Let us consider the closest oriented edge of $C_{\gamma \cdot(2+\omega)}(0)$ to $\bar{\gamma}$, namely, $\ell=(-\omega \cdot \gamma, \bar{\omega} \cdot \gamma)$.
To verify if $\zeta$ and $\zeta^{\prime}$ lay on the left-hand side of $\ell$, we then need to verify the following inequalities

$s^{2}-s t+t<0$,

$s^{2}-s t+s+t<0$,

respectively. Then by substituting $t$ with $s+e$, Inequalities $(13-14)$ are rewritten by

$e(s-1)>s$,

$e(s-1)>2 s$,

respectively. We notice that Inequalities $(15-16)$ are violated when $s=1$ or when $s>1$ and $e=1$.

Finally, we then show that either $\frac{\zeta}{2+\omega}$ or $\frac{\zeta^{\prime}}{2+\omega}$, is an element of $S_{\gamma}^{\prime}(\Lambda, \Lambda)$. First, we have that

$\frac{\zeta}{2+\omega}=-\frac{e+1}{3}+\left(\frac{(1-2 e)}{3}-s\right) \omega$

and

$\frac{\zeta^{\prime}}{2+\omega}=-\frac{e-1}{3}+\left(\frac{2(1-e)}{3}-s\right) \omega$,

where $e=t-s$. We notice that Formula (17) (resp. Formula (18)) has integer values when $e=3 n+$ 2 (resp. $e=3 n+1), n \in \mathbb{Z}$. We note that from Lemma $1, e \not \equiv 0(\bmod 3)$, therefore either $\frac{\zeta}{2+\omega}$ or $\frac{\zeta^{\prime}}{2+\omega}$ has integer values, namely either $\frac{\zeta}{2+\omega} \in \Lambda$ or $\frac{\zeta^{\prime}}{2+\omega} \in \Lambda$.

Finally, since $\operatorname{gcd}(\gamma, \bar{\gamma})=1$, there exist $\kappa \in$ $\Lambda$ and $\lambda \in \Lambda$ such that either $S_{\gamma}^{\prime}(\kappa, \lambda)=\frac{\zeta}{2+\omega}$ or $S_{\gamma}^{\prime}(\kappa, \lambda)=\frac{\zeta^{\prime}}{2+\omega}$.

Lemma 8 If $s=1, t>1$ or $s>0, t=s+1$ we have that $S_{\gamma}^{\prime}(\Lambda, \Lambda) \cap\left(\left(C_{\bar{\gamma}}(0) \cup C_{\gamma}(0)\right) \backslash\left(C_{\bar{\gamma}}(0) \cap C_{\gamma}(0)\right)\right)=$ $\varnothing$.

Proof Thanks to the symmetry of $C_{\gamma \cdot(2+\omega)}(0)$ and $C_{\bar{\gamma} \cdot(2+\omega)}(0)$, we need to focus on a pair of consecutive vertices of $C_{\gamma \cdot(2+\omega)}(0)$, for example, $\gamma$ and $-\omega \cdot \gamma$, and those of $C_{\bar{\gamma} \cdot(2+\omega)}(0)$, (i) $\bar{\gamma}$ and $-\bar{\omega} \cdot \bar{\gamma}$ if $s>0, t=s+1$; (ii) $\omega \cdot \bar{\gamma}$ and $-\bar{\omega} \cdot \bar{\gamma}$ if $s=1, t>0$.

Then we consider the distances between the parallel lines. The pair of parallel lines are defined by (i) $\{\gamma, \bar{\gamma}\}$ and $\{-\omega \cdot \gamma,-\bar{\omega} \cdot \bar{\gamma}\}$; (ii) $\{\gamma,-\bar{\omega} \cdot \bar{\gamma}\}$ and $\{-\omega \cdot \gamma, \omega \cdot \bar{\gamma}\}$ (see Figure 9(a) and (b), respectively). These distances are (i) $d=\frac{1}{2}-\frac{1}{6 s+4}$ and (ii) $d=$ $\frac{\sqrt{3}}{2}-\frac{\sqrt{3}}{2 t+2}$, and thus, (i) $d<\frac{1}{2}$ and (ii) $d<\frac{\sqrt{3}}{2}$. 


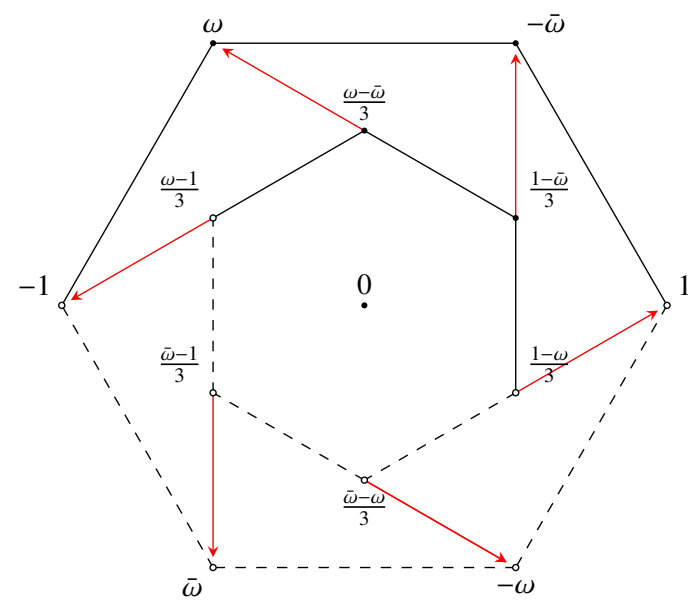

Fig. 7 A unit cell $C_{1}(0)$ and its set of vertices $V$. The outer hexagon is the result of the multiplication $(2+\omega) C_{1}(0)$, namely, $C_{(2+\omega)}(0)$, where the red arrows indicate the new position of the vertices

Since the parallel lines go through points of $\Lambda$ and are (i) parallel and (ii) orthogonal to the hexagonal grid edges, the space between the parallel lines does not contain points of $\Lambda$ - except on the boundary. This implies that $\forall \zeta \in \Lambda, \zeta \in C_{\gamma}(0) \Leftrightarrow \zeta \in$ $C_{\bar{\gamma}}(0)$.

From Lemma 7 and Lemma 8, we obtain the main theorem.

Theorem 1 A digitized rotation associated with $\alpha=\gamma^{2} \in \Lambda_{\rho}, \gamma=(s+t)+t \omega$, is bijective if and only if the generators of $\alpha$ are of the form $s=1, t>0$ or $s>0, t=s+1$.

Even though rational rotations are dense in the hexagonal and the square grids (see Lemma 6), bijective digitized rotations are not dense, as illustrated in Figure 10, where numbers of the biggest bijective angles are presented on the unit circle. Moreover, the asymptotic convergence of the angles are: $\frac{1}{t}$ for the square grid; $\frac{\sqrt{3}}{3 t}$ (for $t=s+1$ ) and $\frac{\sqrt{3}}{t}$ (for $s=1, t>0$ ) for the hexagonal grid. We note that, the limits are multiples of $\frac{\pi}{2}$ and $\frac{\pi}{3}$ for the square and the hexagonal grids, respectively. Note that, in the hexagonal grid case, angles of the family generated by $s=1, t>0$, are asymptotically three times as frequent as the angles given by generators $s>0, t=s+1$. From the frequencies

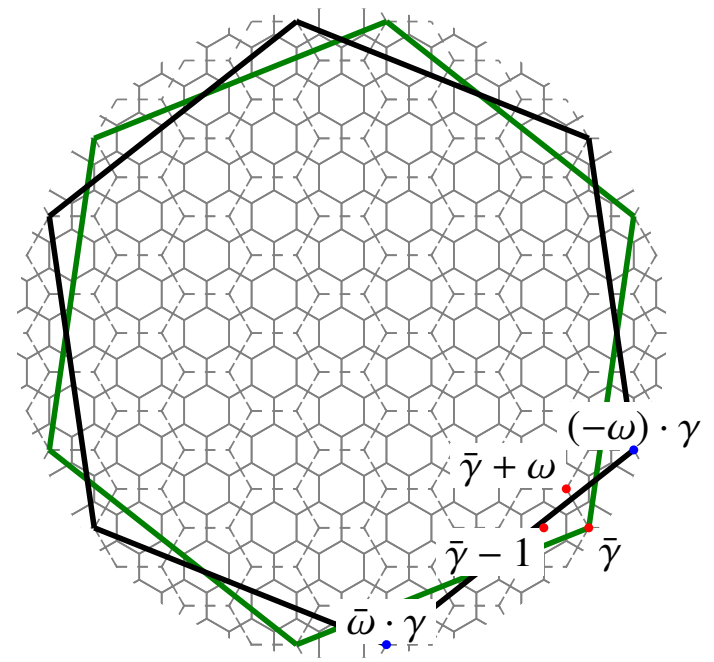

(a)

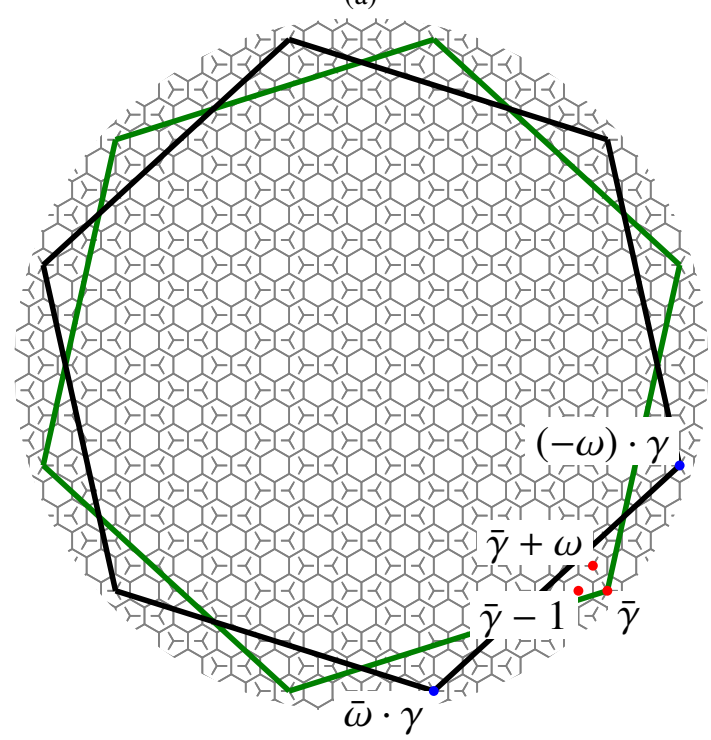

(b)

Fig. 8 Visualization of $C_{\gamma \cdot(2+\omega)}(0)$ and $C_{\bar{\gamma} \cdot(2+\omega)}(0)$ for: $s=$ $3, t=5$ (a); $s=4, t=9$ (b), depicted in black and green, respectively. The usual hexagonal grid is depicted in gray, whereas its mapping by $2+\omega$ is depicted in gray and represented by dashed line segments

we can see that angles for which a bijective rotation exists covers more frequently the unit circle than in the square grid case.

Some examples of bijective and non-bijective digitized rotations on the hexagonal grid are presented in Figure 11. 


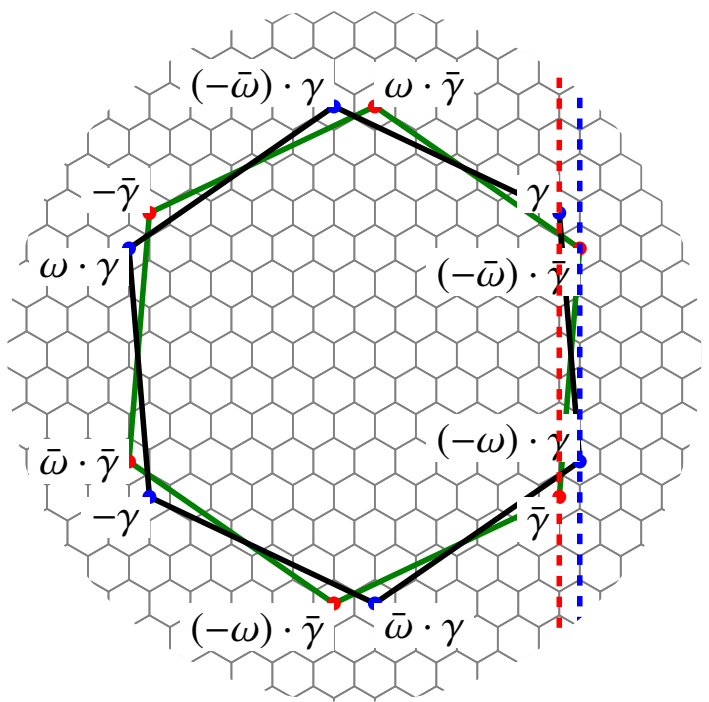

(a)

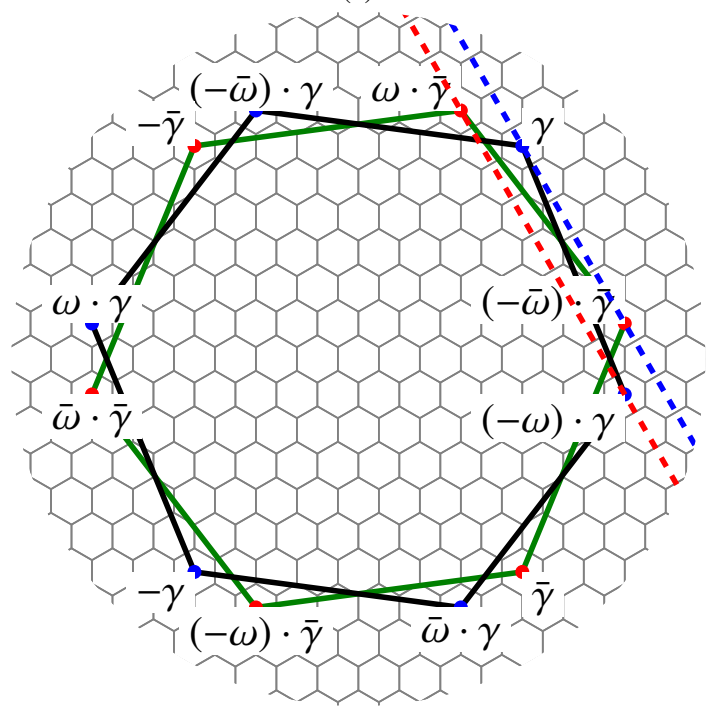

(b)

Fig. 9 Visualization of $C_{\gamma \cdot(2+\omega)}(0)$ and $C_{\bar{\gamma} \cdot(2+\omega)}(0)$ for the case: $s>0, t=s+1$ (a) and $s=1, t>1$ (b). The two parallel lines discussed in the proof of Lemma 8 are illustrated by red and blue dashed lines

\section{Conclusion}

In this article, we characterized bijective digitized rotations defined on the hexagonal grid.

Our approach is similar to that used by Roussillon and Cœurjolly to prove the conditions for bijectivity of $2 \mathrm{D}$ digitized rotations on the square grid using Gaussian integers [23]. In our work, we

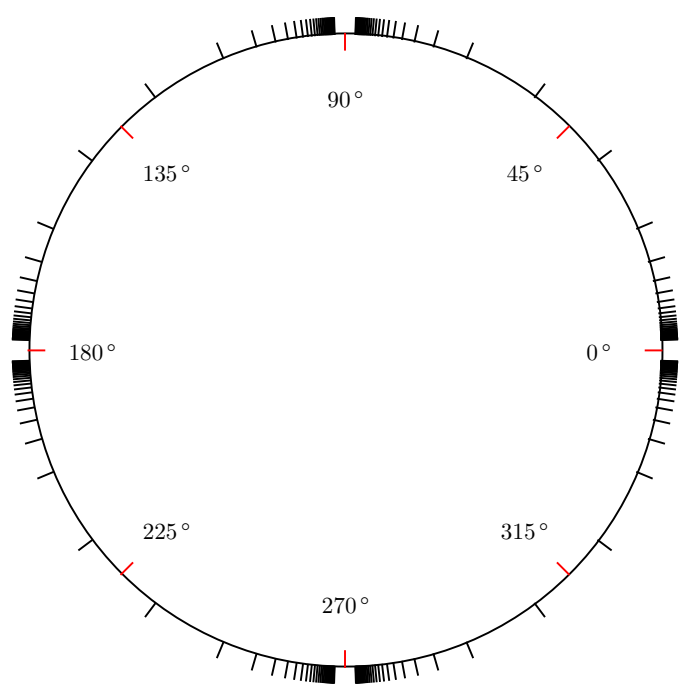

(a)

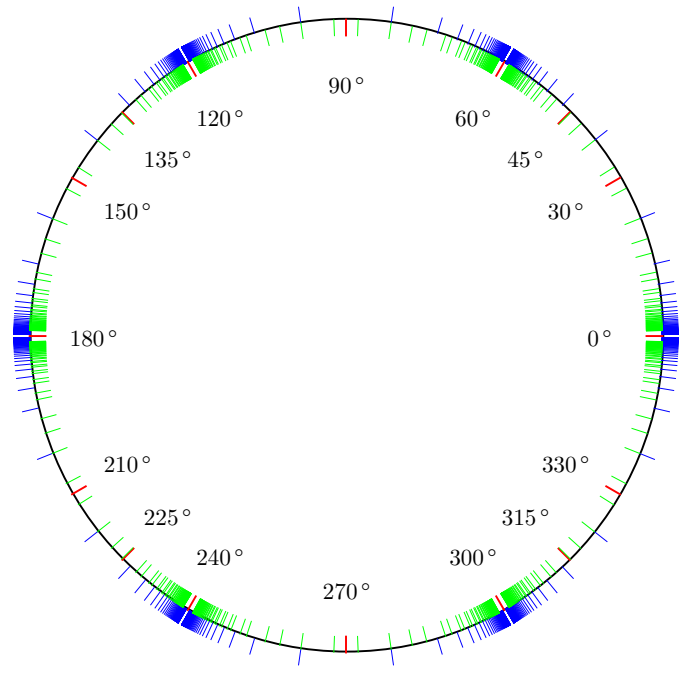

(b)

Fig. 10 Distribution of angles whose digitized rotations by them are bijective in (a) the square and (b) the hexagonal grids. In (b), angles obtain from generators of the form $s>0$ and $t=s+1$ are colored in blue, while angles generated by $s=1$ and $t>0$, are colored in green

used Eisenstein integers, which play a similar role to Gaussian integers. The main difference between bijective digitized rotations defined on the square and the hexagonal grids is that, for the later grid, there exist two families of angles such that respective digitized rotations are bijective. On contrary, there exists only one such a family for digitized ro- 


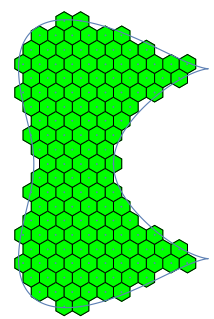

(a)

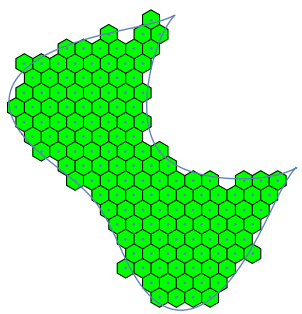

(c)

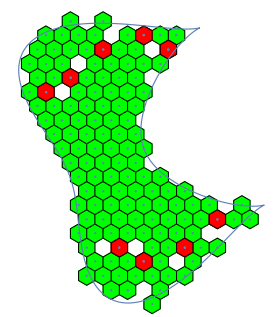

(b)

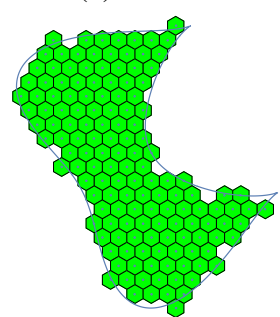

(d)
Fig. 11 Visualization of a bicuspid curve together with Gaussian digitization of its interior on the hexagonal grid (a) and its digitized rotations: non-bijective digitized rotation by angle $\frac{\pi}{9}(\mathrm{~b})$; bijective digitized rotations given by Eisenstein integers generated by $s=1, t=2$ and $s=1, t=3$ (c-d), respectively. In (b) digitization cells which correspond to non-injective cases are marked in red

tations defined on the square grid. In addition, our arithmetical characterization of bijective rational rotations is consistent with the symmetry proprieties of the hexagonal lattice in the sense that angles for which a bijective rotation exists covers more frequently the unit circle than in the square grid case.

\section{References}

1. Anglin, W.S.: Using Pythagorean triangles to approximate angles. American Mathematical Monthly 95(6), 540-541 (1988)

2. Condat, L., Van De Ville, D., Blu, T.: Hexagonal versus orthogonal lattices: A new comparison using approximation theory. In: ICIP 2005, vol. 3, pp. III-1116. IEEE (2005)

3. Condat, L., Ville, D.V.D.: Quasi-interpolating spline models for hexagonally-sampled data. IEEE Trans. Image Processing 16(5), 1195-1206 (2007)

4. Conway, J., Smith, D.: On Quaternions and Octonions. Ak Peters Series. Taylor \& Francis (2003)

5. Fredriksson, K.: Rotation Invariant Template Matching. Ph.D. thesis, University of Helsinki (2001)

6. Gilder, J.: Integer-Sided Triangles with an Angle of $60^{\circ}$. The Mathematical Gazette 66(438), 261-266 (1982)
7. Gordon, R.A.: Properties of Eisenstein triples. Mathematics Magazine 85(1), 12-25 (2012)

8. Hales, T.C.: The honeycomb conjecture. Discrete \& Computational Geometry 25(1), 1-22 (2001)

9. Hardy, G.H., Wright, E.M.: An introduction to the theory of numbers. Oxford University Press (1979)

10. Her, I.: Geometric transformations on the hexagonal grid. IEEE Transactions on Image Processing 4(9), 1213-1222 (1995)

11. Jacob, M.A., Andres, E.: On discrete rotations. In: 5th Int. Workshop on Discrete Geometry for Computer Imagery, pp. 161-174 (1995)

12. Klette, R., Rosenfeld, A.: Digital Geometry: Geometric Methods for Digital Picture Analysis. Elsevier (2004)

13. Kong, T., Rosenfeld, A.: Digital topology: Introduction and survey. Computer Vision, Graphics, and Image Processing 48(3), 357-393 (1989)

14. Middleton, L., Sivaswamy, J.: Edge detection in a hexagonal-image processing framework. Image and Vision Computing 19(14), 1071-1081 (2001)

15. Middleton, L., Sivaswamy, J.: Hexagonal Image Processing: A Practical Approach. Advances in Pattern Recognition. Springer (2005)

16. Nouvel, B., Rémila, E.: On colorations induced by discrete rotations. In: DGCI, Proceedings, Lecture Notes in Computer Science, vol. 2886, pp. 174-183. Springer (2003)

17. Nouvel, B., Rémila, E.: Characterization of bijective discretized rotations. In: R. Klette, J. Žunić (eds.) Combinatorial Image Analysis, Lecture Notes in Computer Science, vol. 3322, pp. 248-259. Springer Berlin Heidelberg (2005)

18. Nouvel, B., Rémila, E.: Configurations induced by discrete rotations: Periodicity and quasi-periodicity properties. Discrete Applied Mathematics 147(2-3), 325-343 (2005)

19. Ostromoukhov, V., Hersch, R.D.: Halftoning by rotating non-bayer dispersed dither arrays. SPIE Milestone Series 154, 238-255 (1999)

20. Ostromoukhov, V., Hersch, R.D., Amidror, I.: Rotated dispersed dither: a new technique for digital halftoning. In: Proceedings of the 21 st annual conference on Computer graphics and interactive techniques, pp. 123-130. ACM (1994)

21. Pluta, K., Romon, P., Kenmochi, Y., Passat, N.: Bijective digitized rigid motions on subsets of the plane. Journal of Mathematical Imaging and Vision (2017)

22. Pluta, K., Romon, P., Kenmochi, Y., Passat, N.: Honeycomb Geometry: Rigid Motions on the Hexagonal Grid. In: DGCI 2017, pp. 33-45. Springer International Publishing, Cham (2017)

23. Roussillon, T., Cœurjolly, D.: Characterization of bijective discretized rotations by Gaussian integers. Research report, LIRIS UMR CNRS 5205 (2016). URL https://hal.archives-ouvertes.fr/hal-01259826

24. Serra, J.: Image Analysis and Mathematical Morphology. Academic Press, London (1982)

25. Yilmaz, A., Javed, O., Shah, M.: Object tracking: A survey. ACM Computing Surveys 38(4) (2006) 